\title{
The Potential of Materials Analysis by Electron Rutherford Backscattering as Illustrated by a Case Study of Mouse Bones and Related Compounds
}

\author{
Maarten Vos, ${ }^{1, \star}$ Károly Tökési, ${ }^{2}$ and Ilona Benkö ${ }^{3}$ \\ ${ }^{1}$ Research School of Physics and Engineering, Australian National University, Canberra, ACT 0200, Australia \\ ${ }^{2}$ Institute of Nuclear Research, Hungarian Academy of Sciences (ATOMKI), P.O. Box 51, H-4001 Debrecen, Hungary \\ ${ }^{3}$ Department of Pharmacology and Pharmacotherapy, Medical and Health Science Center, University of Debrecen, \\ P.O. Box 12, 4032 Debrecen, Hungary
}

\begin{abstract}
Electron Rutherford backscattering (ERBS) is a new technique that could be developed into a tool for materials analysis. Here we try to establish a methodology for the use of ERBS for materials analysis of more complex samples using bone minerals as a test case. For this purpose, we also studied several reference samples containing $\mathrm{Ca}$ : calcium carbonate $\left(\mathrm{CaCO}_{3}\right)$ and hydroxyapatite and mouse bone powder. A very good understanding of the spectra of $\mathrm{CaCO}_{3}$ and hydroxyapatite was obtained. Quantitative interpretation of the bone spectrum is more challenging. A good fit of these spectra is only obtained with the same peak widths as used for the hydroxyapatite sample, if one allows for the presence of impurity atoms with a mass close to that of $\mathrm{Na}$ and $\mathrm{Mg}$. Our conclusion is that a meaningful interpretation of spectra of more complex samples in terms of composition is indeed possible, but only if widths of the peaks contributing to the spectra are known. Knowledge of the peak widths can either be developed by the study of reference samples (as was done here) or potentially be derived from theory.
\end{abstract}

Key words: elastic electron scattering, bone mineral, electron Rutherford backscattering, osteoporosis, elemental analysis, calcium carbonate, hydroxyapatite

\section{INTRODUCTION}

\section{Electron Rutherford Backscattering}

Electron Rutherford backscattering (ERBS) is a novel technique that is being developed at the intersection of electron spectroscopy and ion beam analysis. It is based on the fact that energetic (multiple keV) electrons scattered over large angles transfer a significant amount of momentum to the scattering nucleus (Vos, 2001; Varga et al., 2001, 2006; Went \& Vos, 2007, 2008). As a consequence, the nucleus acquires kinetic energy, and the energy of the electron is reduced by this amount. If an electron beam impinges on a material, consisting of several elements, then the elastically backscattered electrons have an energy distribution consisting of several peaks, each corresponding to scattering from a specific element.

The fractional change in energy is very small, owing to the large mass-mismatch between electrons and nuclei. Hence, a large incoming energy and scattering angle are required (to maximize the momentum transfer) in combination with good energy resolution (better than $0.5 \mathrm{eV}$ full-width at half-maximum) of the spectrometer. This is thus a challenging experiment and explains why this technique is only recently being developed. With one exception [study of thin film $\mathrm{MgB}_{2}$ superconductors (Zhao et al., 2007)], this technique has only been used to study the

Received May 20, 2012; accepted December 29, 2012

*Corresponding author. E-mail: maarten.vos@anu.edu.au physics of the process itself, using samples of well-known composition. Now we want to determine whether ERBS can be used as an analytical technique in a research context. For this, we chose the question of the composition of bone minerals. The mineral content of bones consists of a mixture of elements, in particular $\mathrm{Ca}, \mathrm{P}, \mathrm{Mg}, \mathrm{Na}, \mathrm{O}, \mathrm{C}$, and $\mathrm{H}$, mostly in the form of hydroxyapatite $\left(\mathrm{Ca}_{10}\left(\mathrm{PO}_{4}\right)_{6}(\mathrm{OH})_{2}\right)$, and minor contributions of other phosphates, carbonates, and alkali salts. These elements are all relatively light, and hence their separation in an ERBS spectrum is appreciable. The separation of these elements for our measurement geometry is summarized in Table 1, as well as their differential scattering cross-section calculated using the ELSEPA code (Salvat et al., 2005).

As we will see, the separation of $\mathrm{Ca}$ and $\mathrm{P}$ is less than their intrinsic peak widths. The interpretation of the spectra depends thus on a peak fitting procedure, with the widths of the peaks being a crucial input variable. To get some handle on the Ca peak width, we measure first a calcium carbonate $\left(\mathrm{CaCO}_{3}\right)$ sample. Here the Ca peak stands free, and its area and width can be determined straightforwardly. Next we look at hydroxyapatite, $\mathrm{Ca}_{10}\left(\mathrm{PO}_{4}\right)_{6}(\mathrm{OH})_{2}$, a mineral that is the main constituent of bone mineral, and see how accurately we can obtain the Ca to $\mathrm{P}$ ratio in this case. Finally, we consider mouse bone samples and determine the $\mathrm{Ca}$ to $\mathrm{P}, \mathrm{O}$, and $\mathrm{C}$ ratio, and obtain an estimate of the concentration of sum of the $\mathrm{Na}$ and $\mathrm{Mg}$ atoms present.

In this paper, we mainly focus on the measurement and analysis procedure, and compare finally our best estimate of 
Table 1. Mean Recoil Energy $\left(=\mathbf{q}^{2} / 2 M_{i}\right.$ with $\mathbf{q}$ the Momentum Transfer and $M_{i}$ the Atomic Mass) and Differential Cross-Section (DCS) for the Conditions of the Experiment Described in this Paper $\left(\theta_{\text {scat }}=135^{\circ}, E_{0}=40 \mathrm{keV}\right.$ Corresponding to $|\mathbf{q}|=102 \mathrm{AU}$ or $\left.193 \AA^{-1}\right)$.

\begin{tabular}{lcc}
\hline Element & $\begin{array}{c}E_{\text {rec }} \\
(\mathrm{eV})\end{array}$ & $\begin{array}{c}\mathrm{DCS} \\
\left(\mathrm{cm}^{-2}\right)\end{array}$ \\
\hline $\mathrm{Au}$ & 0.395 & $1.58 \mathrm{E}-20$ \\
$\mathrm{Ca}$ & 1.945 & $4.58 \mathrm{E}-22$ \\
$\mathrm{P}$ & 2.513 & $2.48 \mathrm{E}-22$ \\
$\mathrm{Mg}$ & 3.203 & $1.56 \mathrm{E}-22$ \\
$\mathrm{Na}$ & 3.38 & $1.31 \mathrm{E}-22$ \\
$\mathrm{O}$ & 4.864 & $6.83 \mathrm{E}-23$ \\
$\mathrm{C}$ & 6.485 & $3.81 \mathrm{E}-23$ \\
$\mathrm{H}$ & 77.82 & $1.05 \mathrm{E}-24$ \\
\hline
\end{tabular}

the concentration of the various elements in mouse bone with that obtained by other techniques, as was summarized in a recent paper (Benkö et al., 2012).

\section{Summary of the Theory of ERBS}

For the interpretation of ERBS spectra, one assumes that the trajectory of the detected electron contains only one large-angle scattering event. The large-angle scattering angle $\theta$ can thus be approximated by the angle between the gun and the analyzer. Further, one assumes that the deflection is due to the interaction with the (screened) Coulomb field of a single nucleus, and that this collision between the electron and the nucleus is not affected by the fact that the scattering atom is bound to the lattice. There is, for our present scattering condition (incoming energy $E_{0}=40 \mathrm{keV}, \theta=$ $135^{\circ}$ ), no experimental evidence so far that is at odds with any of these assumptions. Just as in ion Rutherford backscattering, the energy of the scattered electron (mass $m$ ) is reduced after scattering from an atom with mass $M_{i}$ by the familiar kinematic factor $K$ :

$$
K=\frac{E_{1}}{E_{0}}=\frac{m^{2}}{\left(m+M_{i}\right)^{2}}\left\{\cos \theta+\left[\left(\frac{M_{i}}{m}\right)^{2}-\sin ^{2} \theta\right]^{1 / 2}\right\}^{2}
$$

with $E_{1}$ representing the energy of the electron after the scattering event. For electrons scattering from nuclei, this equation can be simplified as $m \ll M_{i}$. We then obtain for the recoil energy loss $E_{\text {rec }}^{i}$ :

$$
E_{\mathrm{rec}}^{i}=(1-K) E_{0} \approx \frac{2 m E_{0}}{M_{i}}(1-\cos \theta) .
$$

In the above, we have neglected relativistic corrections. This is not completely justified under our experimental conditions. Their inclusion adds a factor $\left(1+E_{0} / 2 m c^{2}\right)$ to the right-hand side of equation (2), and this increases the recoil loss at $40 \mathrm{keV}$ by $3.9 \%$.

In the above, we implicitly assumed that the target atom was at rest before the collision. In reality, it vibrates, even at $0 \mathrm{~K}$ (zero-point motion). To see the consequences of this, it is more convenient to analyze the situation in terms of transfered momentum $\mathbf{q}=\mathbf{k}_{0}-\mathbf{k}_{i}$, with $\mathbf{k}_{0}$ and $\mathbf{k}_{1}$ representing the momentum of the electron before and after the collision, respectively. If the momentum of the scattering atom was $\mathbf{p}$ before the collision, then it changes to $\mathbf{p}+\mathbf{q}$ after the collision. As the recoil energy is equal to the change in kinetic energy of the atom, we can write:

$$
E_{\mathrm{rec}}^{i}=\frac{(\mathbf{p}+\mathbf{q})^{2}}{2 M_{i}}-\frac{\mathbf{p}^{2}}{2 M_{i}}=\frac{\mathbf{q}^{2}}{2 M_{i}}+\frac{\mathbf{p} \cdot \mathbf{q}}{M_{i}} .
$$

Thus, the recoil energy is a distribution centered around the recoil energy for scattering from a stationary atom (i.e., $\mathbf{q}^{2} / 2 M_{i}$ ) and has a peak shape that is a Compton profile of the atomic momentum distribution $\mathbf{p}$. In practice, the peak shape is Gaussian and its second moment $\underline{\left(\sigma_{i}\right)}$ can be shown to be related to the mean kinetic energy $\overline{E_{\text {kin }}^{i}}$ of atom $i$ as (see, e.g., Vos et al., 2011):

$$
\sigma_{i}=\sqrt{\frac{4}{3} E_{\mathrm{rec}}^{i} \overline{E_{\mathrm{kin}}^{i}}}
$$

An independent estimate of the quantity $\overline{E_{\text {kin }}^{i}}$ can, in principle, be derived from lattice simulation programs or measured phonon dispersion relations. Here we try to obtain estimates of this width $\sigma_{i}$ (and hence $\overline{E_{\mathrm{kin}}^{i}}$ ) by studying reference samples.

\section{Motivation for the Measurement of the Elemental Composition of Bones}

The human skeleton undergoes a continuous process of remodeling throughout life. The remodeling cycle lasts 6-9 months in healthy adults (Manolagos \& Parfitt, 2010). Bone contains a highly active cellular compartment with a large mineralized matrix. The main elements are calcium and phosphorus, mostly as crystalline hydroxyapatite but also as noncrystalline phosphates and carbonates. More than $99 \%$ of the body's calcium, $80 \%$ of its phosphorus, and $60 \%$ of its magnesium are found in bones. In the extracellular matrix, minerals associated with proteins are synthesized by osteoblasts. These proteins serve as a scaffold for mineralization and they are also responsible for the elasticity of bones (Lakatos \& Takács, 2012). Minerals are important for the strength of bones. In addition, bone plays a role in the overall calcium homeostasis in the body and probably in the homeostasis of some trace elements as well.

Bone maintains its strength through the modulation of its remodeling activity, adapting its structural and material properties in response to its load. A complex control network takes care of the proper bone turnover, and details of the roles of calcium and phosphorus have been studied, but many other trace elements are also regularly found in the extracellular matrix of the bone. However, we do not know much about their importance. Not only calcium but also magnesium deficiency (Rude et al., 2004, 2009; Nielsen, 2010) or high iron content (Guggenbuhl et al., 2008) result in osteoporosis, which suggest that trace elements have some role in mineralization. 
Imbalance in any step of the controlling network may result in osteoporosis. Osteoporosis, characterized by a decrease in bone strength, leads to an increased risk of bone fractures. It constitutes a major public health problem throughout the world as about $10 \%$ of the population is affected. Osteoporosis not only decreases the mineral content of the bones, but also affects the quality of mineralization and disturbs the bone structure (Boivin \& Meunier, 2003). Osteoporosis is defined as a reduction of bone mass or bone density. Little is known about the associated changes in microstructure or distribution of trace elements in the mineral content. This is because only some indirect methods are available to medical practitioners to estimate the mineral content of bones. These noninvasive techniques include dual-energy X-ray absorptiometry (DEXA), singleenergy X-ray absorptiometry, quantitative computed tomography, and ultrasound. DEXA has become the standard method for measuring bone mineral density (BMD). These methods are not able to determine elements separately. Although BMD is believed to be in close association with the risk of osteoporotic fracture, it is not surprising that in many cases fractures appear partially independent of decreased BMD as measured by DEXA (Sarkar et al., 2002; Benhamou, 2007; Sornay-Rendu et al., 2007). A more accurate monitoring of changes in mineral concentrations would be very useful.

A range of spectrometric and microanalytical methods, for example, neutron activation analysis (Saiki et al., 1999; Zaichick \& Tzaphlidou, 2002; Zhang et al., 2002), particleinduced X-ray emission (PIXE) (Warren et al., 2002; Kaabar et al., 2010), and synchrotron radiation-induced X-ray fluorescence (Fei et al., 2007) have been used for elemental mapping, as has the related technique of energy-dispersive $\mathrm{X}$-ray spectroscopy in combination with Auger spectroscopy (Kourkoumelis et al., 2012). Another approach is the Fourier transform infrared spectromicroscopy. It determines the carbonate to phosphate concentration ratio from the relative strength of the infrared absorption features of these groups (Boskey et al., 1998; Amarie et al., 2012). X-ray microtomography has been used to determine the X-ray linear attenuation coefficient from which information on the mineral concentration can be obtained (Davis \& Wong, 1996; Salome et al., 1999). The high intensity available at third-generation synchrotron has been used to do X-ray microscopy of bones with a resolution of $40 \mathrm{~nm}$, and information of elemental composition can be obtained by comparing images above and below the absorption edges (Andrews et al., 2010). Nuclear magnetic resonance techniques are not only able to determine the phosphorus concentration, but also distinguishes between various chemical environments ( $\mathrm{Wu}$ et al., 2002). A comparison of the results of more standard near-surface analytical techniques (XPS, PIXE, and secondary neutral mass spectrometry) was recently given by Benkö et al. (2012).

Backscatter electron (BSE) analysis is used for bone analysis (Bloebaum et al., 1997, 2005; Vajda et al., 1998) and is the technique in bone research that is most closely related to the one described here. BSE analysis also depends on electron backscattering from bones, but all electrons with an energy exceeding a threshold value are counted. The observed count rate then depends on the mean atomic number of the atoms comprising the bones. As the target consists of many elements, the obtained mean atomic number does not allow for the exact sample composition, but the experimental evidence shows that it still provides valuable information of the $\mathrm{Ca} / \mathrm{P}$ concentration ratio. $\mathrm{BSE}$ analysis is a more efficient technique making microscopic visualization of spatially resolved density variations possible. The present technique provides more than a mean atomic number, and the $\mathrm{Ca} / \mathrm{P}$ ratio is derived directly from the experiment, whereas in BSE analysis more indirect indications of the $\mathrm{Ca} / \mathrm{P}$ ratio is obtained by comparing count rate variations in different samples.

Here we present an application of a new direct method to determine the main elements of bone mineral, which could be of use to study pathological changes in animal models or human samples obtained from biopsies. We do not think that this method will replace any of the existing methods, but it could, especially when integrated in an electron microscope be useful as a technique that determines quantitatively the $\mathrm{Ca} / \mathrm{P}$ intensity ratio near the surface.

\section{Materials and Methods}

\section{Experimental Details}

Most of the samples studied here consisted of a fine or somewhat coarse powder. Hydroxyapatite (synthetic; purity, >99.995\%; $\left[\mathrm{Ca}_{5}(\mathrm{OH})\left(\mathrm{PO}_{4}\right)_{3}\right]_{2}$; molecular weight, 502.31; Aldrich Chemical Co.) was studied both as a powder and pressed as a pill. The present study conforms to the European Community guiding principles for care and use of laboratory animals. The experimental protocol was approved by the Hungarian National Ethics Committee for Animal Research (4/2011 DEMAB). Femurs of (Balbc $\times$ DBA) F1 healthy mice (male, 20 weeks old, National Institute of Oncology, Budapest, Hungary) were used. Mice were exterminated by cervical dislocation, and then both femoral bones were removed. They were cleaned from lipids, muscles, and tendons. The bone marrow was washed out with distilled water many times through a fine needle syringe. After this, the bones were dried for a day and were calcinated to prepare bone powder. Calcination (the process of high-temperature heating in the presence of atmospheric oxygen in which all organic material is combusted to $\mathrm{CO}_{2}$ ) was accomplished by burning the bones in a Bunsen burner for $10 \mathrm{~min}$. The end product is pure bone mineral, a compound related to hydroxyapatite, which is very fragile. These bone remnants were broken in a small ceramic mortar. Each mouse bone powder sample contained the femur of five mice. For brevity, we will refer to these samples simply as mouse bone powder.

Ultra-smooth conductive carbon adhesive tabs (ProScitech Pty Ltd., Australia, http://proscitech.com.au) were stuck to a metal shim with an array of 1-mm-diameter holes. 


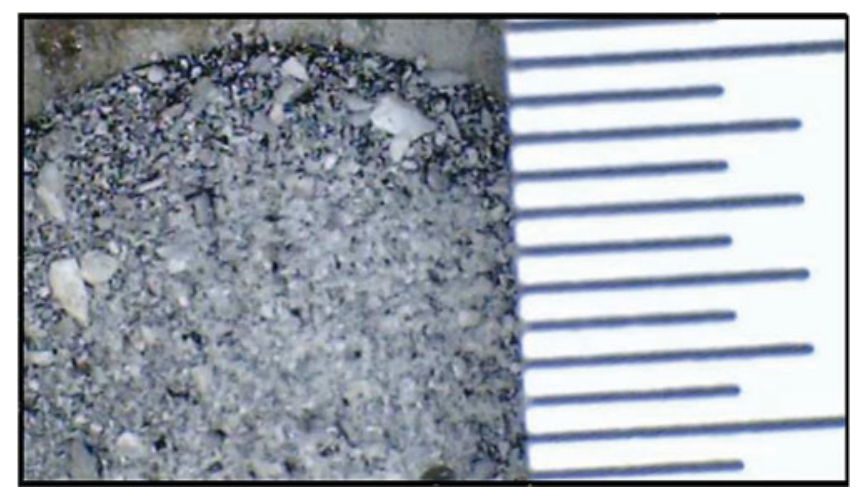

Figure 1. A picture of a part of the 12-mm-diameter tab with the mouse bone stuck to it. A piece of a ruler with $0.5-\mathrm{mm}$ division is shown as well.

The powder was pressed on the tabs filling the holes in the steal shim. The coarser mouse bone pieces were pressed directly on the tab, without a shim being present (see Fig. 1).

An outline of the spectrometer is shown in Figure 2. The method was described in some detail in Went and Vos (2008). Since then, the scattering angle was increased to $135^{\circ}$ and the two-dimensional detector readout was changed from a resistive anode to a phosphor screen camera combination. The first change slightly improved the separation of the peaks, owing to increased momentum transfer. The second change improved the energy resolution somewhat, in particular it removed almost all of the non-Gaussian wings that could be seen in the elastic peak shape when using a resistive anode as the detector.

The $0.2-\mathrm{mm}$ beam was aligned with the holes in the shim. Well-covered holes were selected. Moreover, it was checked that the beam was well away from the metal shim.

Often, a quick scan was made first to ascertain that no heavy elements were present in the ERBS spectrum. For the actual measurement, we subsequently evaporated $\approx 1 \AA \mathrm{Au}$ on the surface. As we will see, Au has, due to its large mass, a very sharp elastic peak. The Au signal thus provides us with an internal check of the actual spectrometer resolution during the measurement. The true zero-energy loss position of the spectrometer is also easily obtained from the sharp Au elastic peak, which for $E_{0}=40 \mathrm{keV}$ is at an energy loss of $0.395 \mathrm{eV}$. In practice, the Au peak was found slightly away from this value, as the zero point of the energy scale of the spectrometer depends somewhat on the sample position. In the figures presented here, a small quantity is added or subtracted to the energy scale, in such a way that the $\mathrm{Au}$ peak always appears at $0.395 \mathrm{eV}$.

Typically, a current of $\approx 7 \mathrm{nA}$ was used during the measurement. No change of the spectra with electron beam exposure was observed. A single measurement took typically $2 \mathrm{~h}$, and the measurement was repeated for three different sample positions.

The scattered electrons were decelerated and analyzed for energy using a hemispherical electrostatic analyzer. The peak width of the Au elastic peak was $\approx 0.5 \mathrm{eV}$ full-width at half-maximum.

\section{Data Analysis}

Under the present experimental conditions, all indications are that the contribution of an element to an ERBS spectrum can be represented by a Gaussian centered at a recoil energy loss of $E_{\text {rec }}^{i}$. As explained before, there is a small uncertainty in the zero point of the energy scale. Another quantity that is somewhat unknown is the incoming energy. This is mainly due to the fact that the samples studied here are insulators, and it is possible that the surface charges as a consequence of the small current of impinging high-energy electrons. For example, if the sample charges by $1 \mathrm{keV}$, then the experiment would effectively be done with $39 \mathrm{keV}$ impinging electrons and the actual recoil energy would be $39 / 40 \times E_{\mathrm{rec}}^{i}$.

In light of these uncertainties, the spectrum is fitted by the following formula:

$$
\begin{aligned}
I(E)= & y_{0}+\sum_{i} \frac{A_{i}}{\sqrt{2 \pi\left(\sigma_{i}^{2}+\sigma_{\mathrm{res}}^{2}\right)}} \\
& \times e^{-\left(E-E_{\mathrm{offset}}-\kappa E_{\mathrm{rec}}^{i}\right)^{2} /\left(2 \sigma_{i}^{2}+2 \sigma_{\mathrm{res}}^{2}\right)},
\end{aligned}
$$

where the parameters are $y_{0}$ an energy-independent background (due to detector "dark" count rate), $E_{\text {offset }}$ a small shift due to the uncertainty in the true zero loss position in the spectrum, $\kappa$ a parameter, close to 1 , describing the effect of possible charging of the sample, $\sigma_{i}$ is the intrinsic width of component $i$, i.e., the Doppler broadening of this component, as described by equation (3). This variable is often kept constant, as will be explained later. $\sigma_{\text {res }}$ is the resolution of the spectrometer. From measurements at $45^{\circ}$ scattering angle of $\mathrm{Au}$, where we are sure that the Doppler broadening is small, we judge the resolution $\sigma_{\text {res }}$ to be $0.14 \mathrm{eV}$ (i.e., $0.35 \mathrm{eV}$ full-width at half-maximum). A different choice of the experimental resolution will result in different intrinsic width of the different components, but will not affect the ratio of their intensities. $A_{i}$ is the area of component $i$.

In the fitting procedure, $E_{\text {rec }}^{i}$ is always kept fixed at the mean recoil energy $q^{2} / 2 M_{i}$, and the experimental resolution $\sigma_{\text {res }}$ is set to a value such that the intrinsic width of $\mathrm{Au}$ $\left(\sigma_{\mathrm{Au}}\right)$, the sharpest peak, is $0.14 \mathrm{eV}$. This is the intrinsic width calculated for Au using equation (4), assuming that $\overline{E_{\text {kin }}}=\frac{3}{2} k T$. The latter assumption should be reasonable for $\mathrm{Au}$ as its Debeye temperature $(170 \mathrm{~K})$ is considerable less than the measurement temperature $(293 \mathrm{~K})$. The observed dependence of the full-width at half-maximum of the $\mathrm{Au}$ peak on the scattering angle $\left(0.35 \mathrm{eV}\right.$ for $\theta=45^{\circ} ; 0.5 \mathrm{eV}$ for $\theta=135^{\circ}$ ) is consistent with the assumption that for $\mathrm{Au}$ $\overline{E_{\mathrm{kin}}}=\frac{3}{2} k T$. The values of the parameters that were not fixed are obtained by minimizing the $\chi^{2}$.

For the other elements contributing to our spectrum, we have no reason to assume that $\overline{E_{\text {kin }}}=\frac{3}{2} k T$. Indeed, we will see that their kinetic energies are often significantly larger. In principle, one could calculate their kinetic energies from the crystal structure in combination with a proper interatomic potential. Currently, this is not done routinely. Hence, 


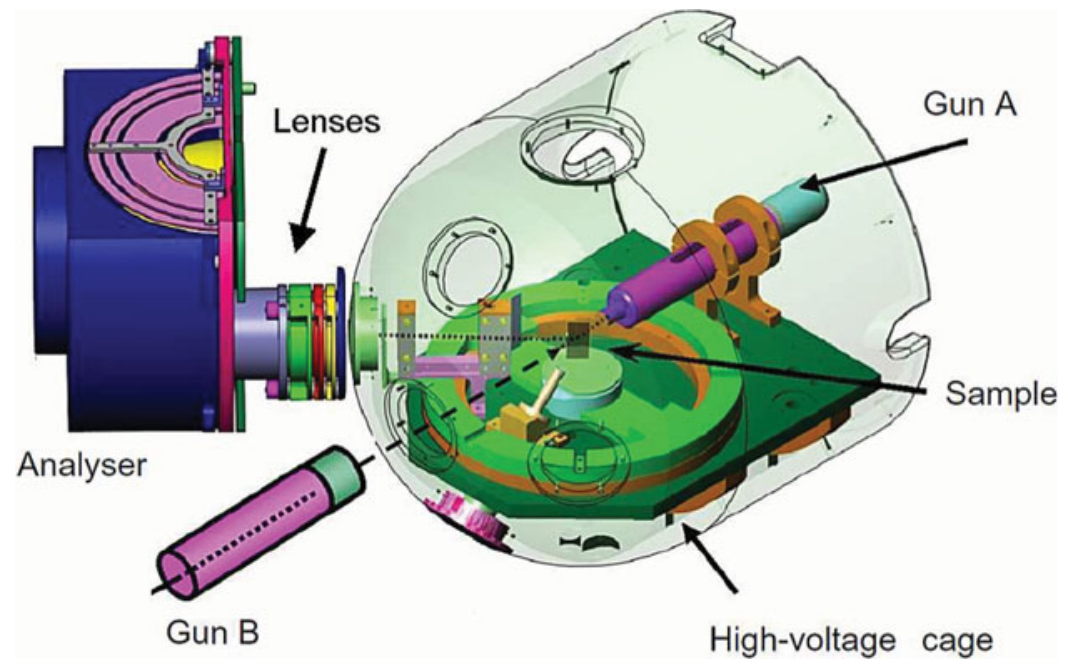

Figure 2. A schematic overview of the spectrometer. In this work, gun B is used, which produces $500 \mathrm{eV}$ electrons. The sample is in a high-voltage area, kept at $39.5 \mathrm{keV}$. Thus, $40 \mathrm{keV}$ electrons hit the sample, and we detect electrons scattered over $135^{\circ}$. These electrons are first decelerated to $\approx 200 \mathrm{eV}$ and then analyzed for energy by an electrostatic analyzer. we follow a somewhat different route. We first measure $\mathrm{CaCO}_{3}$, where the $\mathrm{Ca}$ is well resolved. Subsequently, we study hydroxyapatite, a mineral of known composition, closely related to the constituent of bones, and determine whether we can analyze this sample using either the width as a free parameter or using the same width for $\mathrm{Ca}$ as in $\mathrm{CaCO}_{3}$. Next we analyze the mouse bone sample, again considering the widths of $\mathrm{Ca}$ and $\mathrm{P}$ as free parameters, or using the $\mathrm{P}$ and $\mathrm{Ca}$ width as obtained for hydroxyapatite.

\section{Results}

We discuss the results for different samples in order of increasing complexity. Our experience with the "simple" cases is used to unravel the more complex cases.

\section{Results for $\mathrm{CaCO}_{3}$}

We start with $\mathrm{CaCO}_{3}$, where the $\mathrm{Ca}$ peak is not expected to overlap with any other contributions to the elastic region of the spectrum. A sample with a $\mathrm{CaCO}_{3}$ powder was introduced into the vacuum chamber. It was measured as is, and the low-loss region of the energy loss spectrum showed two clear peaks, and a shoulder at higher energy losses (see Fig. 3). On the basis of the calculated recoil losses and atomic elastic scattering cross-sections (see Table 1), we interpret the peak at lowest energy loss to be due to electrons scattered elastically from $\mathrm{Ca}$, the second due to electrons scattered from $\mathrm{O}$, and the shoulder due to electrons scattered from C. Clearly, the Ca peak is sharper than the $\mathrm{O}$ peak and $\mathrm{C}$ shoulder. To make the interpretation even more rigorous, we evaporated $\approx 1 \AA$ of Au onto the sample surface.

After Au evaporation, the ERBS spectrum showed three peaks and a shoulder, as shown in the main panel of Figure 3. The new peak, attributed to $\mathrm{Au}$, is at lower energy loss than the peak attributed to $\mathrm{Ca}$, and is even narrower than the Ca peak. Thus, most of the width of the Ca peak is intrinsic, i.e., the vibrational motion of the atom causes Doppler broadening of the elastic peak [see equation (4)]. Owing to the large scattering cross-section of Au, even an

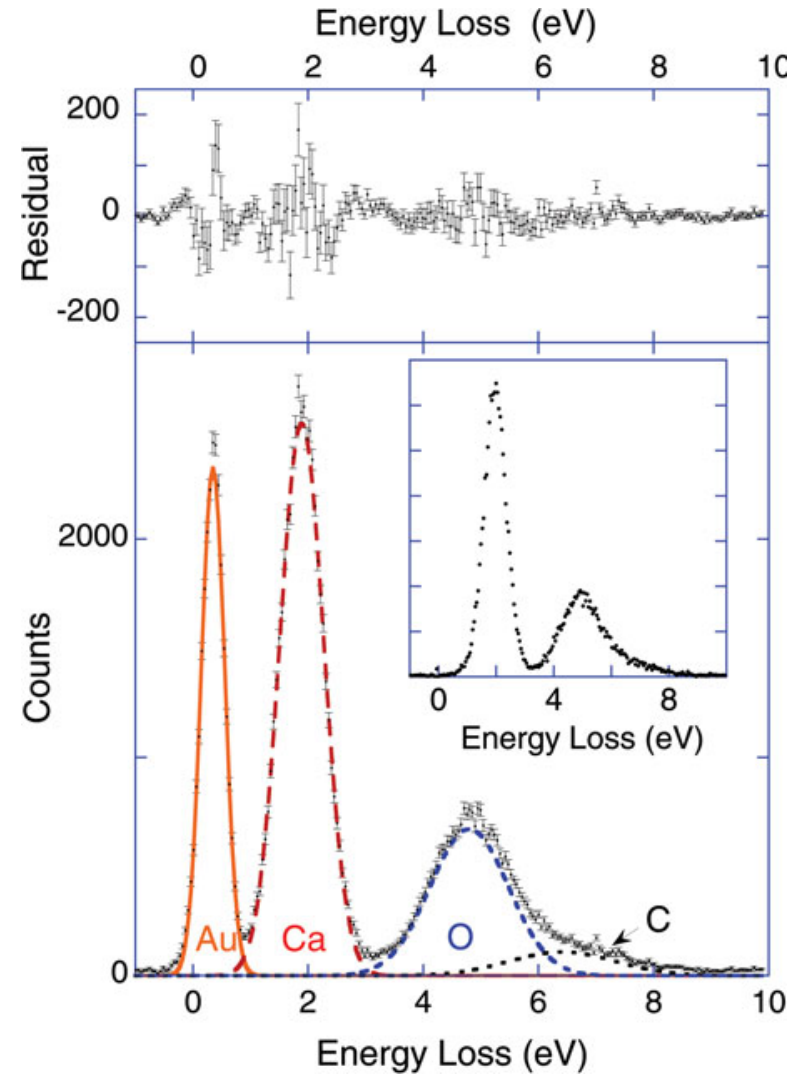

Figure 3. The decomposition of the electron Rutherford backscattering spectrum of $\mathrm{CaCO}_{3}$ with a small amount of Au evaporated at the surface in the various components. The top panel shows the residual of the fit. The inset shows a spectrum of $\mathrm{CaCO}_{3}$ before $\mathrm{Au}$ deposition.

$\approx 1 \AA$ thin layer contributes an appreciable peak to the spectrum. This does not imply, however, that ERBS is extremely surface-sensitive (Vos \& Went, 2007). The depth probed is of the order of the inelastic mean free path, which, according to the TPP2M formula, is $512 \AA$ for $40 \mathrm{keV}$ electrons in $\mathrm{CaCO}_{3}$ (Tanuma et al., 1994).

This spectrum of $\mathrm{CaCO}_{3}$ with $\mathrm{Au}$ was fitted using equation (5), as well as the spectra obtained from two other 
Table 2. Fitting Parameters (Peak Width and Peak Intensity Ratios) of the $\mathrm{CaCO}_{3}$ Plus Au Spectra for Three Measurements at Different Spots of the Sample.

\begin{tabular}{lcrccc}
\hline Sample & $I_{\mathrm{Ca}} / I_{\mathrm{O}}$ & $I_{\mathrm{Ca}} / I_{\mathrm{C}}$ & $\begin{array}{c}\sigma_{\mathrm{Ca}} \\
(\mathrm{eV})\end{array}$ & $\begin{array}{c}\sigma_{\mathrm{O}} \\
(\mathrm{eV})\end{array}$ & $\begin{array}{c}\sigma_{\mathrm{C}} \\
(\mathrm{eV})\end{array}$ \\
\hline$\# 1$ & 2.11 & 9.71 & 0.355 & 0.665 & 0.910 \\
$\# 2$ & 2.14 & 9.52 & 0.356 & 0.682 & 0.942 \\
$\# 3$ & 2.08 & 9.62 & 0.355 & 0.675 & 0.957 \\
Calculated & 2.23 & 12.02 & - & - & - \\
\hline
\end{tabular}

spots on the same sample. A good fit was obtained with a value for $\kappa$ of $0.996 \pm 0.002$. Nominally, this corresponds to charging of the sample by $160 \mathrm{~V}$, but such a small deviation of $\kappa$ (corresponding to a peak separation change of $\mathrm{Au}$ and $\mathrm{C}$ of $0.02 \mathrm{eV}$ ) could also be caused by calibration errors of either the high-voltage power supply or the positionsensitive detector. The result indicates that charging of the sample is limited and is not a factor that would make ERBS hard to apply to this kind of poorly conducting samples. These results also show that the peak separation is in very good agreement with the simple (and purely classical) theory of electrons scattering from free atoms.

The result of the fitting is further summarized in Table 2. The reproducibility of the Ca:O intensity ratio is good, with the lowest and highest ratio differing by only $3 \%$. The Ca:C intensity ratio is more challenging to determine accurately, as the $\mathrm{C}$ peak is only observed as a shoulder. Nevertheless, the lowest and highest value differ by only a few percent. It is even more interesting to compare these intensity ratios with the theoretically calculated ones, using the calculated elastic scattering cross-sections given in Table 1 . The Ca:O intensity ratio obtained in this way is only $4-7 \%$ larger than the observed one. For the $\mathrm{Ca}: \mathrm{C}$ ratio, the agreement is not as good, with the area of the C peak 23-25\% larger than expected. This probably indicates the existence of different contributions at these energy losses (such as scattering from a $\mathrm{Ca}$ atom plus the creation of an electronic excitation), which are not negligibly small compared to the weak intensity of electrons scattered by carbon. Finally, using equation (4), the measured width of $\mathrm{Ca}, \mathrm{O}$, and $\mathrm{C}$ corresponds to the mean kinetic energy of the atoms of, respectively, $0.049 \mathrm{eV}$, $0.070 \mathrm{eV}$, and $0.101 \mathrm{eV}$, all significantly more than $\frac{3}{2} k T$ $(=0.0375 \mathrm{eV})$.

\section{Results for Hydroxyapatite}

Encouraged by the good results for $\mathrm{CaCO}_{3}$ we now proceed with hydroxyapatite $\left(\mathrm{Ca}_{10}\left(\mathrm{PO}_{4}\right)_{6}(\mathrm{OH})_{2}\right)$, on which again $\approx 1 \AA$ of Au was evaporated. In principle, ERBS can detect hydrogen as well (Vos, 2002; Yubero et al., 2005; Yubero \& Tökèsi, 2009), but because of its low concentration in hydroxyapatite, low cross-section (see Table 1), and presumably large peak width, hydrogen detection is not realistic in the present case, and the measured spectra was restricted to the low energy-loss region.

One set of measurements was done on hydroxyapatite powder and one set on hydroxyapatite pressed in a pill.
Table 3. The Result of the Measurement of Hydroxyapatite for Three Different Spots Using a Thick (T) Sample and a Powder (P) Sample, as well as the Calculated Ratios.

\begin{tabular}{lccccc}
\hline Sample & $I_{\mathrm{Ca}} / I_{\mathrm{P}}$ & $I_{\mathrm{Ca}} / I_{\mathrm{O}}$ & $\begin{array}{c}\sigma_{\mathrm{Ca}} \\
(\mathrm{eV})\end{array}$ & $\begin{array}{c}\sigma_{\mathrm{P}} \\
(\mathrm{eV})\end{array}$ & $\begin{array}{c}\sigma_{\mathrm{O}} \\
(\mathrm{eV})\end{array}$ \\
\hline $\mathrm{T} \# 1$ & 2.83 & 2.39 & 0.336 & 0.512 & 0.644 \\
$\mathrm{~T} \# 2$ & 3.33 & 2.36 & 0.353 & 0.486 & 0.697 \\
$\mathrm{~T} \# 3$ & 3.25 & 2.35 & 0.353 & 0.484 & 0.710 \\
$\mathrm{P} \# 1$ & 2.87 & 2.45 & 0.348 & 0.504 & 0.659 \\
$\mathrm{P} \# 2$ & 4.08 & 2.68 & 0.381 & 0.547 & 0.693 \\
P \#3 & 3.23 & 2.42 & 0.355 & 0.496 & 0.691 \\
Calculated & 3.08 & 2.58 & - & - & - \\
\hline
\end{tabular}

At first glance, the obtained spectra look rather similar to those of $\mathrm{CaCO}_{3}$ plus $\mathrm{Au}$ (see Fig. 4), but fitting the spectra with the $\mathrm{Au}, \mathrm{Ca}$, and $\mathrm{O}$ elastic peaks gives a fit that is much poorer than the fit in the $\mathrm{CaCO}_{3}$ case.

After including the $\mathrm{P}$ elastic peak in the fit, the result, shown in Figure 5, is of comparable quality to the fits of the $\mathrm{CaCO}_{3}$ spectra. Thus, the phosphorus peak, although not resolved, has to be included in the fitting procedure to obtain a good description of the experimental result. The parameters obtained by the fitting procedure are summarized in Table 3. Now the variations in the Ca:P intensity ratios obtained appear quite large: up to $40 \%$ for the extremes. (Excluding measurement $\mathrm{P} \# 2$ the variations are $<17 \%$.). Clearly, there is a strong correlation between the fitting parameters of the overlapping peaks, in particular the peak width and peak area parameters. The large width obtained for the $\mathrm{Ca}$ component in measurement $\mathrm{P} \# 2$ results in a large area for the Ca peak, and hence to a large $\mathrm{Ca}: \mathrm{P}$ peak intensity ratio. The situation is somewhat better for the Ca:O intensity ratio. Here the observed ratios are within $12 \%$.

The variation in the observed ratios becomes much less if we keep the width of each component fixed. We reanalyzed the measurement with a fixed width corresponding to the average value of all six measurements in Table 3. For $\mathrm{Ca}$, the average intrinsic width was $0.353 \mathrm{eV}$, which is very close to the value obtained for $\mathrm{CaCO}_{3}(0.355 \mathrm{eV})$. This agreement is encouraging and could indicate that such a width is typical for $\mathrm{Ca}$ in an ionic environment, but this agreement could also be accidental. If such patterns exist, they will become apparent when more data become available. When using the value of the peak width obtained by averaging over all measurements, then the variations in $\mathrm{Ca}: \mathrm{P}$ intensity ratio is considerable less $(10 \%$ between the extremes, see Table 4) and for the $\mathrm{Ca}: \mathrm{O}$ intensity ratio the range is $8 \%$. Agreement between the calculated Ca:P intensity ratio (3.08) and the measurement (average of the experimental ratio is 3.16) is surprisingly good and the experimental values scatter around the calculated one. The theoretically expected $\mathrm{Ca}: \mathrm{O}$ intensity ratio is slightly outside the range of the measurement, but the agreement between all measurements and the calculated value is better than $10 \%$ 


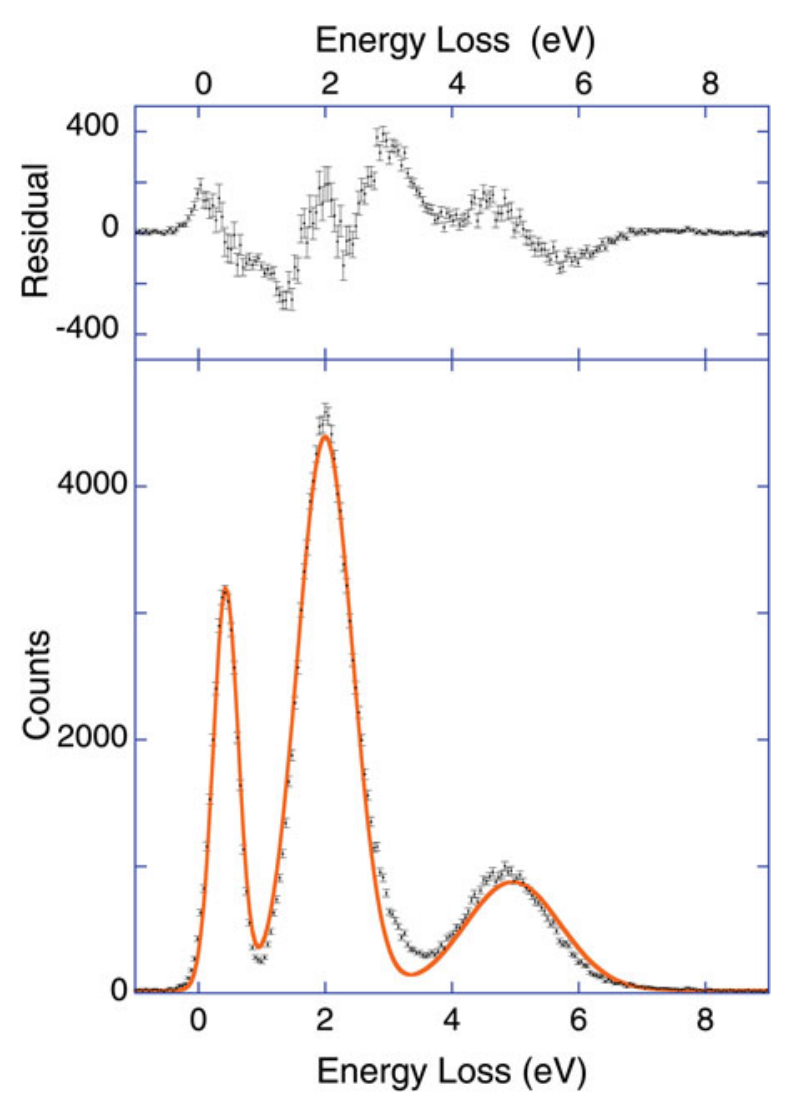

Figure 4. An attempt to fit the hydroxyapatite electron Rutherford backscattering spectrum after $\mathrm{Au}$ evaporation with $\mathrm{Au}, \mathrm{Ca}$, and $\mathrm{O}$. The quality of the fit is much less than that of the $\mathrm{CaCO}_{3}+\mathrm{Au}$ spectrum shown in Figure 3.

\section{Results for Mouse Bone Powder}

The bone samples consisted of a a much coarser powder than either $\mathrm{CaCO}_{3}$ or apatite samples. A picture of the bone particles pressed on a 12 -mm-diameter tab is shown in Figure 1. The beam spot is $\approx 0.25 \mathrm{~mm}$ diameter, only slightly larger than the average particle size of the bone fragments. Therefore, the spectrum is obtained from only a few grains. There is a slight variation in the color of the grains. Thus, the sample is not uniform and some variability can be expected for measurements of such samples. The

Table 4. The Result of the Intensity Ratios for Hydroxyapatite Obtained from the Same Measurements as in Table 3, but now with the Width Kept Fixed at the Average Value Obtained from All Measurements $\left(\sigma_{\mathrm{Ca}}=0.353 \mathrm{eV}, \sigma_{\mathrm{P}}=0.505 \mathrm{eV}, \sigma_{\mathrm{O}}=0.683 \mathrm{eV}\right)$.

\begin{tabular}{lcc}
\hline Sample & $I_{\mathrm{Ca}} / I_{\mathrm{P}}$ & $I_{\mathrm{Ca}} / I_{\mathrm{O}}$ \\
\hline $\mathrm{T} \# 1$ & 3.28 & 2.40 \\
$\mathrm{~T} \# 2$ & 3.22 & 2.40 \\
$\mathrm{~T} \# 3$ & 3.08 & 2.39 \\
$\mathrm{P} \# 1$ & 3.00 & 2.45 \\
$\mathrm{P} \# 2$ & 3.34 & 2.55 \\
$\mathrm{P} \# 3$ & 3.07 & 2.43 \\
Calculated & 3.08 & 2.58 \\
\hline
\end{tabular}

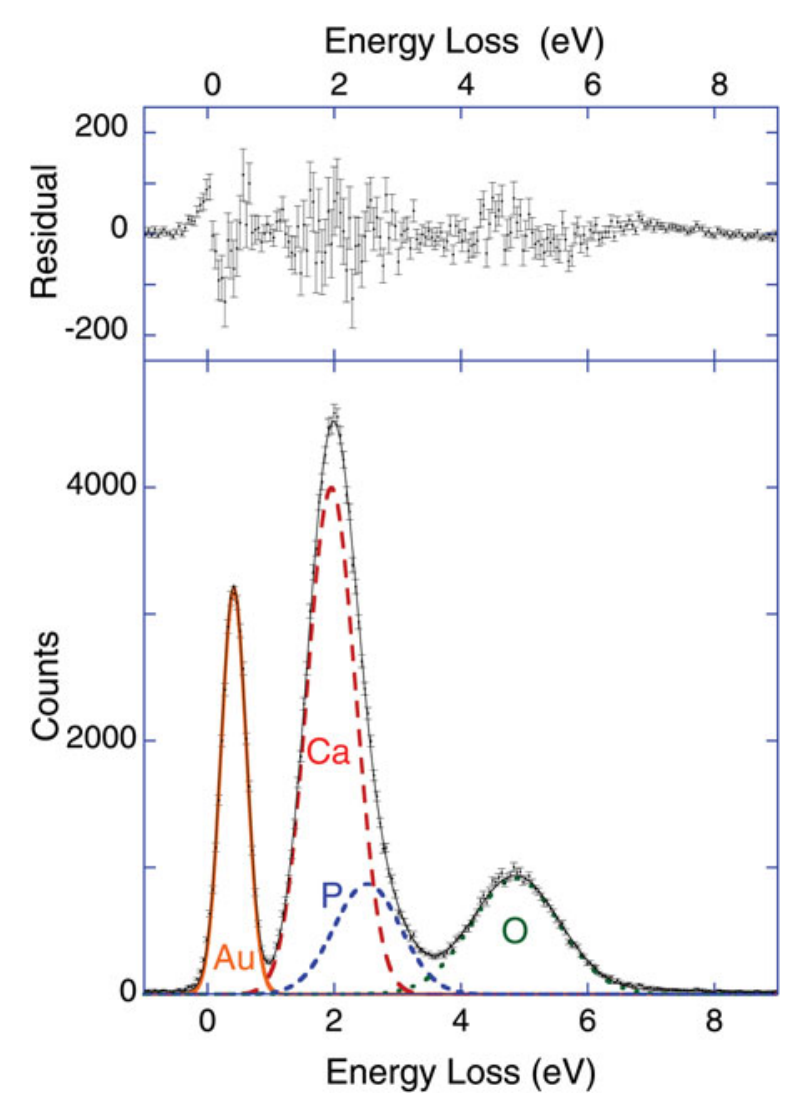

Figure 5. A fit of the hydroxyapatite $(+\mathrm{Au})$ spectrum with components for $\mathrm{Au}, \mathrm{Ca}, \mathrm{P}$, and $\mathrm{O}$.

possibility that the carbon backing contributes slightly to the spectra cannot be excluded.

Again spectra were obtained from three different spots after $\approx 1 \AA$ Au was evaporated on the sample. One spectrum showed, even without fitting, clearly more carbon than the other two spectra. First, the spectra were fitted with all widths treated as free variables (Table 5). Good fits were obtained, as can be seen by the example shown in Figure 6 . However, the width of especially the $\mathrm{P}$ peak is considerable larger than in the "parent" mineral hydroxyapatite $(\sigma \approx 0.57 \mathrm{eV}$ in mouse bone versus $0.505 \mathrm{eV}$ in hydroxyapatite). One would not expect the width of any component to depend on details of the sample composition. Hence, we re-fitted the spectrum with the same width for $\mathrm{Ca}$, $\mathrm{P}$, and $\mathrm{O}$ as in hydroxyapatite, and (more speculatively) the same width of $\mathrm{C}$ as that of $\mathrm{C}$ in $\mathrm{CaCO}_{3}$. Now the quality of the fit was somewhat less good, as can be seen in Figure 7 .

Table 5. The Results of the Fitting of the Mouse Bone Spectra Treating the Peak Width as a Fitting Parameter.

\begin{tabular}{lccccccc}
\hline Sample & $I_{\mathrm{Ca}} / I_{\mathrm{P}}$ & $I_{\mathrm{Ca}} / I_{\mathrm{O}}$ & $I_{\mathrm{Ca}} / I_{\mathrm{C}}$ & $\begin{array}{c}\sigma_{\mathrm{Ca}} \\
(\mathrm{eV})\end{array}$ & $\begin{array}{c}\sigma_{\mathrm{P}} \\
(\mathrm{eV})\end{array}$ & $\begin{array}{c}\sigma_{\mathrm{O}} \\
(\mathrm{eV})\end{array}$ & $\begin{array}{c}\sigma_{\mathrm{C}} \\
(\mathrm{eV})\end{array}$ \\
\hline$\# 1$ & 2.07 & 1.89 & 6.37 & 0.344 & 0.581 & 0.686 & 0.92 \\
$\# 2$ & 2.03 & 1.95 & 18.3 & 0.365 & 0.58 & 0.699 & 0.87 \\
$\# 3$ & 2.09 & 2.03 & 20.0 & 0.345 & 0.546 & 0.677 & 0.97 \\
\hline
\end{tabular}




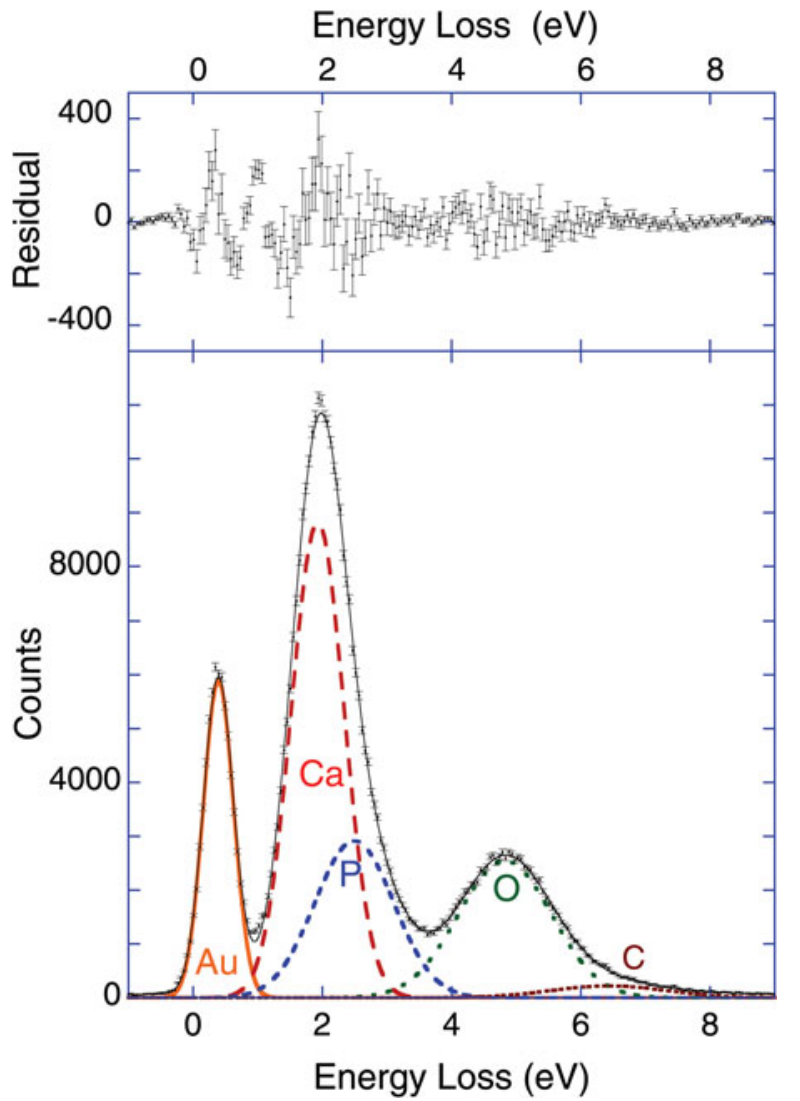

Figure 6. A fit of a mouse bone spectrum with all widths treated as free-fitting parameters.

There is clearly some intensity missing near $3.3 \mathrm{eV}$, and the fitting procedure reduces the $\kappa$ parameter somewhat to fill this hole, resulting in a slight misalignment of the $\mathrm{O}$ peak.

From the literature (Benkö et al., 2012), we know that there are minor quantities of $\mathrm{Mg}$ and $\mathrm{Na}$ in mouse bone. As is clear from Table 1, the elastic peak of both elements will contribute near $3.3 \mathrm{eV}$, as their recoil losses (and their scattering cross-sections) are similar. We have rather arbitrarily chosen to add a peak for the element Mg to the fitting procedure, but the area associated with this peak should be considered to be due to the sum of the amount of $\mathrm{Mg}$ and $\mathrm{Na}$ present in the sample. For this reason, we refer to this component as "Mg" rather than $\mathrm{Mg}$. By including this extra component (while keeping the width of the major components fixed, as described before), the fit improves considerably (see Fig. 8). Moreover, the relative P intensity decreases somewhat (see Table 6). The observed Ca:P ratio for the mouse bone sample is now close to the values found for hydroxyapatite. The measured values of Benkö et al. (2012) for the Ca:P ratio of bone samples $\left[n_{\mathrm{Ca}} / n_{\mathrm{P}}=1.86\right.$ based on PIXE and $n_{\mathrm{Ca}} / n_{\mathrm{P}}=1.8$ based on X-ray photoelectron spectroscopy (XPS)] are rather similar to that of hydroxyapatite where the Ca:P ratio is 1.66 . Our result for mouse bone (average peak area ratio $I_{\mathrm{Ca}} / I_{\mathrm{P}}=2.65$ ) corresponds to a concentration ratio of $n_{\mathrm{Ca}} / n_{\mathrm{P}}=1.43$. Without inclusion of the "Mg" component, we have an average value of $I_{\mathrm{Ca}} / I_{\mathrm{P}}=2.06$,

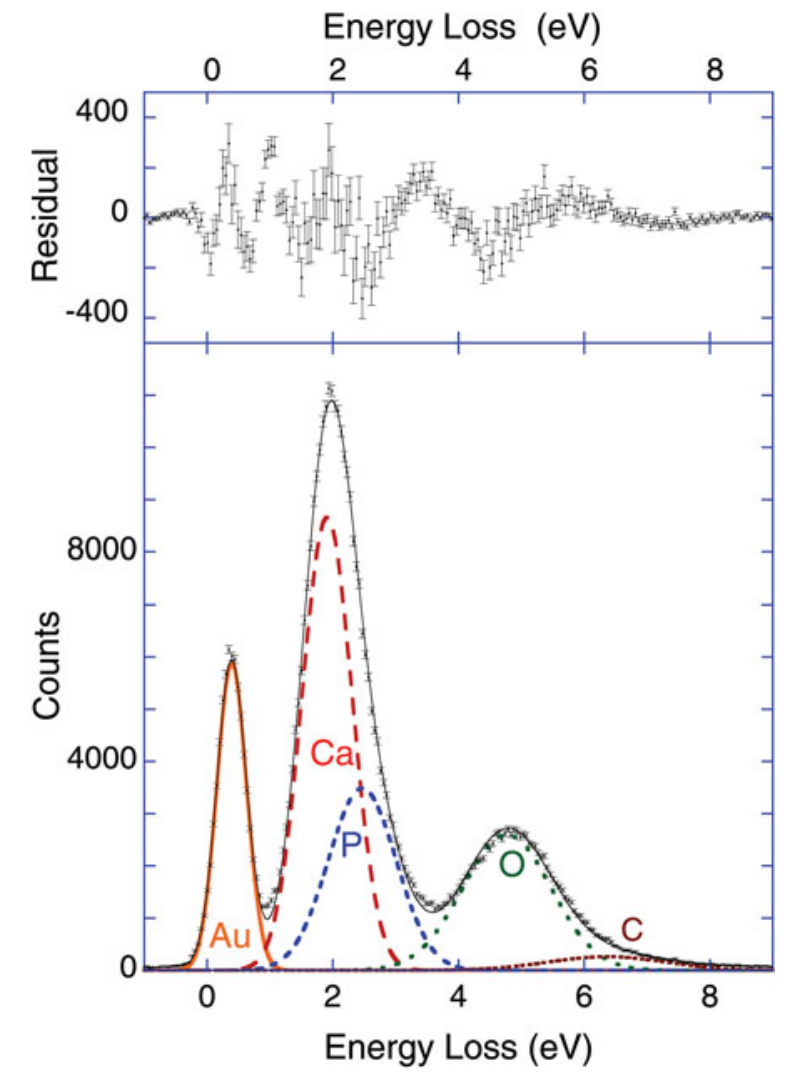

Figure 7. A fit of a mouse bone spectrum with all widths fixed to the corresponding values in hydroxyapatite and $\mathrm{CaCO}_{3}$. Note that the residual plot now shows considerably more structure above $2 \mathrm{eV}$ energy loss than the fit in Figure 6.

which corresponds to $n_{\mathrm{Ca}} / n_{\mathrm{P}}=1.11$, even more at odds with the literature data.

From the average ratio of the area of the "Mg" and Ca component $\left(I_{\mathrm{Ca}} / I^{\prime \prime} \mathrm{Mg}\right.$ " $\left.=19.7\right)$ and their cross-sections, we conclude that the $n_{\mathrm{Ca}} / n^{\prime \prime M g "}=6.7$. In Benkö et al. (2012), one obtains from the XPS data a value of $n_{\mathrm{Ca}} / n_{\mathrm{Mg}}=12.7$. The XPS experiment resolves $\mathrm{Mg}$ and $\mathrm{Na}$, and a similar quantity of $\mathrm{Na}$ is visible in their spectra compared with $\mathrm{Mg}$. Thus, our estimate of the combined concentration of $\mathrm{Mg}$ and $\mathrm{Na}$ is in line with the XPS data. We want to stress that this ERBS measurement of the combined $\mathrm{Mg}$ plus $\mathrm{Na}$ concentration in mouse bone should be treated as somewhat speculative, as we have no experience with the interpretation of ERBS data at this level of precision.

Table 6. The Result of the Intensity Ratios for Mouse Bone Obtained from the Same Measurements as in Table 5, But Now with the Width Kept Fixed on the Average Value Obtained from Hydroxyapatite and $\mathrm{CaCO}_{3}\left(\sigma_{\mathrm{Ca}}=0.353 \mathrm{eV}, \sigma_{\mathrm{P}}=0.505 \mathrm{eV}, \sigma_{\mathrm{O}}=\right.$ $0.683 \mathrm{eV}, \sigma_{\mathrm{C}}=0.93 \mathrm{eV}$ ). Also, Atoms with the Mass of $\mathrm{Mg}$, Were Added to the Fit, as Described in the Main Text.

\begin{tabular}{lcccc}
\hline Sample & $I_{\mathrm{Ca}} / I_{\mathrm{P}}$ & $I_{\mathrm{Ca}} / I_{\mathrm{O}}$ & $I_{\mathrm{Ca}} / I_{\mathrm{C}}$ & $I_{\mathrm{Ca}} / I_{\text {“Mg” }}$ \\
\hline$\# 1$ & 2.92 & 2.04 & 6.7 & 16.1 \\
$\# 2$ & 2.32 & 2.02 & 16.0 & 20.4 \\
$\# 3$ & 2.70 & 2.13 & 22.4 & 22.6 \\
\hline
\end{tabular}




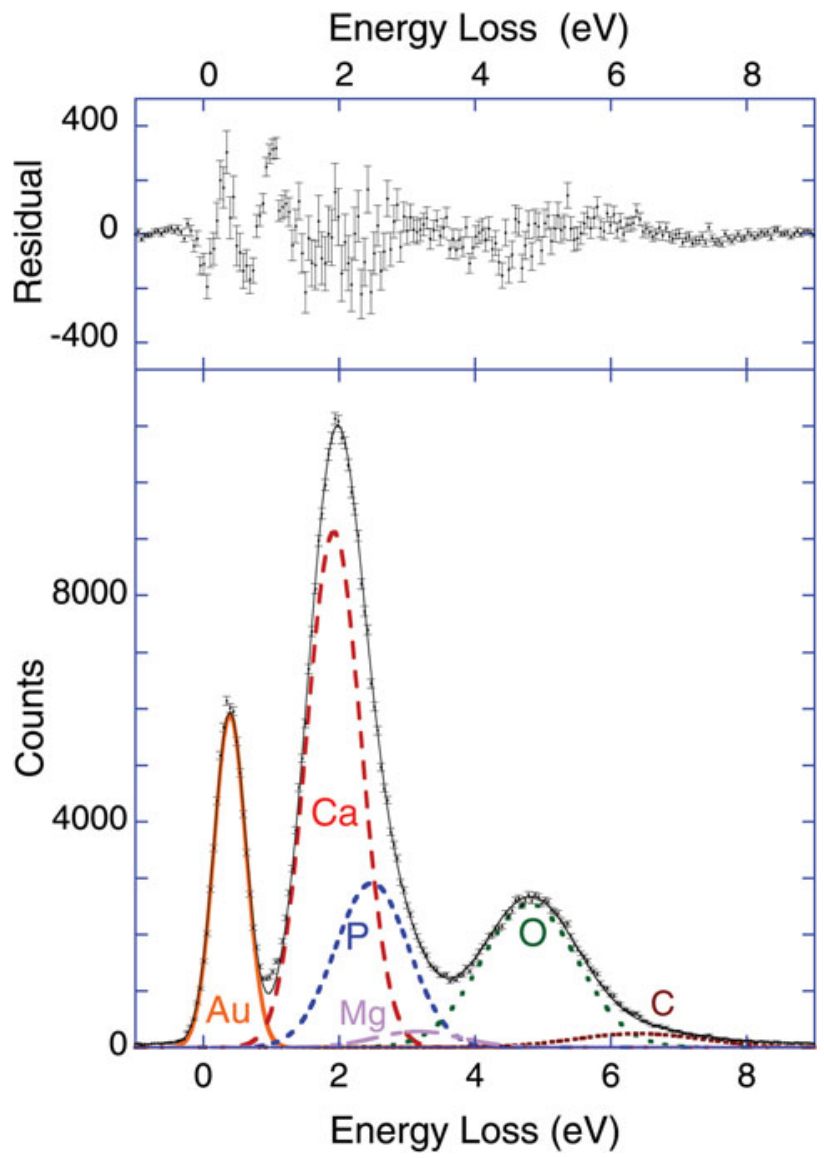

Figure 8. A fit of a mouse bone spectrum with all widths fixed to the corresponding values in hydroxyapatite and $\mathrm{CaCO}_{3}$, but now with one more component corresponding to $\mathrm{Mg}$. The width of the $\mathrm{Mg}$ component $\left(\sigma_{\mathrm{Mg}}\right)$ was set to $0.6 \mathrm{eV}$

The fact that inclusion of an "Mg" component in the model has quite an influence on the value of $I_{\mathrm{Ca}} / I_{\mathrm{P}}$ illustrates well that we have to treat the numbers obtained here with some caution. Assuming that only a limited number of elements are present, then the fitting procedure gives the concentration of these elements. The statistics of the measurement are good enough to determine the $\mathrm{Ca} / \mathrm{P}$ ratio with an accuracy of better than 5\%, provided that the fitting function used is $100 \%$ correct. In reality, our fitting function is incomplete (e.g., iron is present in bones but not included in the fit), resulting in a much larger absolute uncertainty.

On the other hand, the evidence for the phosphorus concentration is much more direct than in either DEXA, where conclusions are reached about the $\mathrm{P}$ concentration based on the difference of X-ray absorption at (only two) different energies, or BSE, which basically uses the intensity of the energy-integrated ERBS spectrum as an indication of the $\mathrm{P}$ concentration. Thus, ERBS should currently be seen as a semi-quantitative technique that determines the elemental concentration relatively close $(\approx 25 \mathrm{~nm})$ to the surface, and as always it will be easier (and often more useful) to establish trends between different samples rather than the absolute concentration.

\section{Discussion}

ERBS has a number of distinct advantages for semiquantitative analysis of samples. For example, in contrast to XPS:

- Its high energy makes it rather insensitive to surface effects.

- The peaks appear well approximated by Gaussian with no obvious problems due to background subtraction.

- All the electrons involved have very similar kinetic energy, and hence one does not need to consider changes of the mean-free path or the analyzer transmission function.

- There are no intrinsic satellites to complicate the quantitative interpretation.

On the other hand, ERBS has a weak point, peaks tend to overlap. The peak width is, especially for the lighter elements, an intrinsic property, and for these elements the situation cannot be improved by increasing the energy resolution. The situation increases slowly if one increases the incoming electron energy (while keeping the scattering angle constant). Peak separation is proportional to the incoming energy $E_{0}$, but the intrinsic peak width increases proportional to the square root of $E_{0}$.

In practice, one has to live with overlapping peaks. An important conclusion of the work, as described here, is that quantitative analysis is often still possible, but only if one has some knowledge of which elements could be present in the sample and their peak width.

With increased experience one will get well-founded expectations for the peak width of each element in a sample. These expectations can either be based on reference samples or on calculated width, based on the lattice vibrational properties of a material. Thus, there is a clear prospect that for samples, mainly composed of low and medium $Z$ elements, ERBS can be developed in a competitive technique for the determination of their (near-surface) composition.

Another point of concern is how accurate the calculated elastic scattering cross-sections are. The calculations used (Salvat et al., 2005) are considered "state-of-the-art", and the underlying physics appears reasonably well established, but cross-sections at large scattering angles have only been verified at much lower energies $(<1 \mathrm{keV})$ for a selected number of elements (noble gases and certain high-vapour pressure metals) (Salvat, 2003). The calculations included the effect of exchange, polarization, and absorption, but only the last contribution is significant. The level of consistency obtained here can be seen as confirmation of the theory under these conditions. If the concentration of some elements is systematically under- or overestimated by ERBS, then this could be interpreted as sign of complications in the cross-section calculations, but no clear trends have emerged so far.

An earlier attempt to measure the composition of materials using ERBS was somewhat less successful (Went \& Vos, 2007). In that study, we used single crystals. Since then, we have learned that for single crystals diffraction effects 
can change the intensity ratio of the different components by a large amount (Winkelmann \& Vos, 2011). The current powder samples should not be affected by this complication.

\section{CONCLUSION}

The promise of ERBS as an analytical technique is clear from the successful analysis of the "simple case" of $\mathrm{CaCO}_{3}$ and the somewhat more complicated case of hydroxyapatite. Here agreement between the measured and actual composition are on a very respectable $10 \%$ level. The actual bone sample showed more variation in outcome and a somewhat less satisfactory agreement between experiment and theory. The variation in outcome between the different measurements can be due to the fact that we average over very few grains, and that the grain composition will vary somewhat with its original position in the bone. In summary, ERBS can become a useful tool to study bone mineralization. As our knowledge of bone mineralization is incomplete, this is currently an active topic of multidisciplinary research involving people working in physics, chemistry, and biological/medical sciences. In general, this case study has shown that ERBS provides an interesting new flavor of microanalysis, a technique that, we think, could have interesting applications, especially if it is integrated in some kind of electron microscopy.

\section{ACKNOWLEDGMENTS}

This research was made possible by a grant of the Australian Research Council and the Medical and Health Research Council of the Hungarian government (ETT293/2009). The authors want to thank Prof. Erich Weigold for fruitful discussions and critical reading of the manuscript.

\section{REFERENCES}

Amarie, S., Zaslansky, P., Kajihara, Y., Griesshaber, E., Schmahl, W.W. \& Keilmann, F. (2012). Nano-FTIR chemical mapping of minerals in biological materials. Beilstein J Nanotechnol 3, 312-323.

Andrews, J.C., Almeida, E., Van Der Meulen, M.C., Alwood, J.S., Lee, C., Liu, Y., Chen, J., Meirer, F., Feser, M., Gelb, J., Rudati, J., Tкachuk, A., Yun, W. \& Pianetta, P. (2010). Nanoscale X-ray microscopic imaging of mammalian mineralized tissue. Microsc Microanal 16, 327-336.

Benhamou, C. (2007). Effects of osteoporosis medications on bone quality. Joint Bone Spine 74, 39-47.

Benkö, I., Rajta, I., Csik, A., Tóth, J., Benkö, K., Géresi, K., Ungvári, E., Szabó, B., Sarkadi, G., Paripás, B., Takács, I. \& TöKÉsı, K. (2012). Major and trace elements in mouse bone measured by surface and bulk sensitive methods. Nucl Instrum and Meth B 279, 223-226.

Bloebaum, R.D., Holmes, J.L. \& Skedros, J.G. (2005). Mineral content changes in bone associated with damage induced by the electron beam. Scanning 27, 240-248.

Bloebaum, R., Skedros, J., Vajda, E., Bachus, K. \& Constantz, B. (1997). Determining mineral content variations in bone using backscattered electron imaging. Bone 20, 485-490.
Boivin, G. \& Meunier, P. (2003). The mineralization of bone tissue: A forgotten dimension in osteoporosis research. Osteopor Int 4(Suppl 3), S19-S24.

Boskey, A., Gadaleta, S., Gundberg, C., Doty, S., Ducy, P. \& Karsenty, G. (1998). Fourier transform infrared microspectroscopic analysis of bones of osteocalcin-deficient mice provides insight into the function of osteocalcin. Bone 23, 187-196.

Davis, G.R. \& Wong, F.S.L. (1996). X-ray microtomography of bones and teeth. Physiol Meas 17, 121-146.

Fei, Y., Zhang, M., Li, M., Huang, Y., He, W., Ding, W. \& Yang, J. (2007). Element analysis in femur of diabetic osteoporosis model by SRXRF microprobe. Micron 38, 637-642.

Guggenbuhl, P., Filmon, R., Mabilleau, G., Baslé, M. \& ChapPARD, D. (2008). Iron inhibits hydroxyapatite crystal growth in vitro. Metabolism 57, 903-910.

Kaabar, W., Gundogdu, O., Laklouk, A., Bunk, O., Pfeiffer, F., Farquharson, M. \& Bradley, D. (2010). Micro-PIXE and SAXS studies at the bone-cartilage interface. Appl Radiat Isot 68, 730-734.

Kourkoumelis, N., Balatsoukas, I. \& Tzaphlidou, M. (2012). $\mathrm{Ca} / \mathrm{P}$ concentration ratio at different sites of normal and osteoporotic rabbit bones evaluated by Auger and energy dispersive X-ray spectroscopy. J Biol Phys 38, 279-291.

Lakatos, P. \& Takács, I. (2012). Metabolic Bone Disorders. Budapest, Hungary: Semmelweis Press.

Manolagos, S. \& Parfitt, A. (2010). What old means to bone. Trends Endocrinol Metab 21, 369-374.

Nielsen, F. (2010). Magnesium inflammation, and obesity in chronic disease. Nutr Rev 68, 333-340.

Rude, R., Gruber, H., Norton, H., Wei, L., Frausto, A. \& Mills, B. (2004). Bone loss induced by dietary magnesium reduction to $10 \%$ of the nutrient requirement in rats is associated with increased release of substance $\mathrm{p}$ and tumor necrosis factoralpha. J Nutr 134, 79-85.

Rude, R., Singer, F. \& Gruber, H. (2009). Skeletal and hormonal effects of magnesium deficiency. J Am Coll Nutr 28, 131-141.

Saiki, M., Takata, M., Kramarski, S. \& Borelli, A. (1999). Instrumental neutron activation analysis of rib bone samples and bone reference materials. Biol Trace Elem Res 71-72, 41-46.

Salome, M., Peyrin, F., Cloetens, P., Odet, C., Laval-Jeantet, A.M., Baruchel, J. \& Spanne, P. (1999). A synchrotron radiation microtomography system for the analysis of trabecular bone samples. Med Phys 26, 2194-2204.

Salvat, F. (2003). Optical-model potential for electron and positron elastic scattering by atoms. Phys Rev A 68, 012708-1-17.

Salvat, F., Jablonski, A. \& Powell, C.J. (2005). ElSEPA Dirac partial-wave calculation of elastic scattering of electrons and positrons by atoms, positive ions and molecules. Comput Phys Commun 165, 157-190.

Sarkar, S., Mitlak, B., Wong, M., Stock, J., Black, D. \& Harper, K. (2002). Relationships between bone mineral density and incident vertebral fracture risk with raloxifene therapy. J Bone Miner Res 17, 1-10.

Sornay-Rendu, E., Munoz, F., Delmas, P. \& Chapurlat, R. (2007). The FRAX tool in french women: How well does it describe the real incidence of fracture in the OFELY cohort? $J$ Bone Miner Res 25, 2101-2107.

Tanuma, S., Powell, C.J. \& Penn, D.R. (1994). Calculations of electron inelastic mean free paths. V. data for 14 organic compounds over the 50-2000 eV range. Surf Interface Anal 21, 165-176.

Vajda, E.G., Skedros, J.G. \& Bloebaum, R.D. (1998). Errors in quantitative backscattered electron analysis of bone standard- 
ized by energy-dispersive X-ray spectrometry. Scanning 20, 527-535.

Varga, D., Tökési, K., Berényi, Z., Tóth, J., Körvér, L., Gergely, G. \& Sulyok, A. (2001). Energy shift and broadening of the spectra of electrons backscattered elastically from solid surfaces. Surf Interface Anal 31, 1019-1026.

Varga, D., Tokési, K., Berényi, Z., Tóth, J. \& Kövér, L. (2006). Observation of the hydrogen peak in the spectra of electrons backscattered from polyethylene. Surf Interface Anal 38, 544-547.

Vos, M. (2001). Observing atom motion by electron-atom Compton scattering. Phys Rev A 65, 12703-1-5.

Vos, M. (2002). Detection of hydrogen by electron Rutherford backscattering. Ultramicroscopy 92, 143-149.

Vos, M., Moreh, R. \& Tökési, K. (2011). The use of electron scattering for studying atomic momentum distributions: The case of graphite and diamond. J Chem Phys 135, 024504-1-7.

Vos, M. \& Went, M.R. (2007). Experimental confirmation of the EPES sampling depth paradox for overlayer/substrate systems. Surf Sci 601, 1536-1543.

Warren, M., Kravchenko, A.F.I., Dunnam, F., Rinsvelt, H.V. \& Maples, W. (2002). Elemental analysis of bone: Proton-induced $\mathrm{X}$-ray emission testing in forensic cases. Forensic Sci Int 125, $37-41$.

Went, M. \& Vos, M. (2007). Investigation of binary compounds using electron Rutherford back scattering. Appl Phys Lett 90, 072104-1-3.

Went, M. \& Vos, M. (2008). Rutherford backscattering using electrons as projectiles: Underlying principles and possible applications. Nucl Instrum Methods Phys Res B 266, 998-1011.
Winkelmann, A. \& Vos, M. (2011). Site-specific recoil diffraction of backscattered electrons in crystals. Phys Rev Lett 106, 085503-1-4.

Wu, Y., Ackerman, J.L., Kim, H.-M., Rey, C., Barroug, A. \& Glimcher, M.J. (2002). Nuclear magnetic resonance spin-spin relaxation of the crystals of bone, dental enamel, and synthetic hydroxyapatites. J Bone Miner Res 17, 472-480.

Yubero, F., Rico, V.J., Espinos, J.P., Cotrino, J. \& GonzalezElipe, A.R. (2005). Quantification of the H content in diamondlike carbon and polymeric thin films by reflection electron energy loss spectroscopy. Appl Phys Lett 87, 084101-1-3.

Yubero, F. \& Tökèsı, K. (2009). Identification of hydrogen and deuterium at the surface of water ice by reflection electron energy loss spectroscopy. Appl Phys Lett 95, 084101-1-3.

Zaichick, V. \& Tzaphlidou, M. (2002). Determination of calcium, phosphorus and the calcium/phosphorus ratio in cortical bone from the human femoral neck by neutron activation analysis. Appl Radiat Isot 56, 781-786.

Zhang, Y., Li, D., Wang, Y., Zhuang, G., Cheng, F., Zhang, G., WANG, Z. \& XIA, J. (2002). Investigation of elemental distribution in iliac crests of female New Zealand rabbits using NAA. Biol Trace Elem Res 86, 65-72.

Zhao, Y., Wu, Y., Kong, C., Wexler, D., Vos, M., Went, M. \& Dou, S.X. (2007). Phase evolution in PLD MgB2 films during the in situ annealing process. Supercond Sci Technol 20, S467-S471. 